\title{
COPPER NANOPARTICLES COATING ON FILTER USING BIOLOGICAL METHOD AND THE STUDY OF ITS ANTIBACTERIAL ACTIVITY
}

\author{
Nima BANIJAMALI ${ }^{\mathrm{a}}$ and Hamid Reza GHORBANI ${ }^{\mathrm{b}, *}$ \\ ${ }^{a}$ Department of Chemistry, Pharmaceutical Sciences Branch, Islamic Azad University, Tehran, Iran \\ ${ }^{\mathrm{b}}$ Department of Chemical Engineering, Qaemshahr Branch, Islamic Azad University, Qaemshahr, Iran
}

In present study, the copper nanoparticles were coated on the surface of water purification filter using a biological technique. The coating characteristics were investigated using X-ray diffraction (XRD), transmission electron microscopy (TEM), Uv-vis spectroscopy and atomic absorption spectroscopy (AAS). The antimicrobial activity of the prepared filter was studied by measuring the diameter of the inhibition zone. In this work, two bacteria: one gramnegative of Escherichia coli and one gram-positive Staphylococcus aureus were used for antibacterial

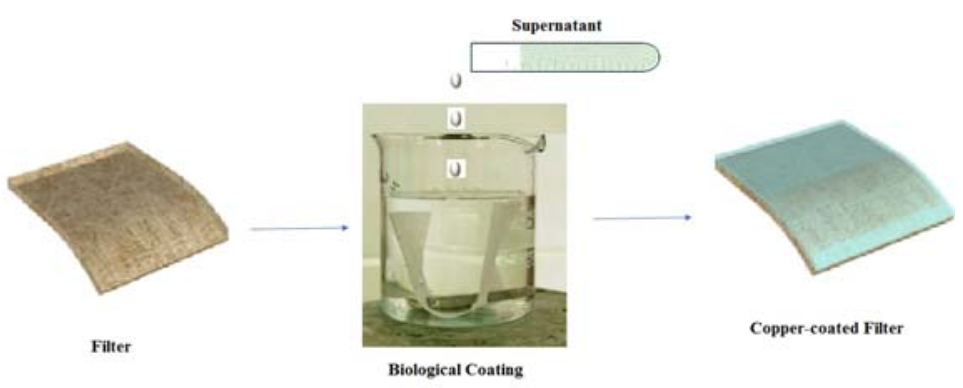
analysis, and it was determined that coated filter exhibited an appropriate antibacterial activity against these two bacteria.

\section{INTRODUCTION}

Nanotechnology is an interesting field nowadays, involving in synthesis, design, characterization, production and application of structures, by controlling shape and size at the nanometer scale. The past decade has seen significant interest directed towards the synthesis of metal nanoparticles due to their unique physicochemical properties and their potential applications in various fields, which include biological labelling, catalysis, diagnostics, electronics, and sensor technology. ${ }^{1}$ The techniques used to produce nanoparticles can be classified as physicochemical or biological. Many physicochemical synthetic methods have been devised, but they are often expensive and involve the use of toxic or hazardous chemicals that pose potential environmental and biological risks. ${ }^{2}$ Therefore, biological processes based on the use of microorganisms or plants have been considered as possible means for synthesizing nanoparticles, especially "green" synthetic processes. ${ }^{3}$ Furthermore, the nanoparticles formed by biological methods are stable for a long time whereas those produced using chemical methods are unstable and tend to aggregate. ${ }^{4}$ Accordingly, biological entities, such as, microorganisms (algae, bacteria, fungi, and yeast) and plants have been investigated for the synthesis of copper nanoparticles. ${ }^{3,4}$ Of the many possible biological sources, microorganisms are preferred for biosynthesis due to their processing speeds, ease of culture, and downstream processing and manipulation considerations. $^{5}$ In addition, the synthesis of copper nanoparticles using microorganisms does not require the laborious extraction processes needed for processing plant biomasses. The microbial synthesis of copper nanoparticles can take place either intracellularly or extracellularly. Copper nanoparti-

\footnotetext{
*Corresponding author: hamidghorbani6@gmail.com
} 
cles have wide applications in heat transfer fluids, sensors, ${ }^{6,7}$ catalysis, solar energy, antibacterial and antifungal materials and batteries. ${ }^{3,8}$ Escherichia Coli is the most important members of the Enterobacteriaceae, which includes close races between them. Escherichia is equally matched genetically with Shigella. This family is part of the normal flora as endemic naturally in the gut of humans and other animals. Facultative anaerobic bacteria, which are gram negative rod, grow anaerobically and produce acids and gas.

Although, the application of biological systems to synthesis of copper nanoparticles has a history of several decades, the mechanisms responsible for copper nanoparticles synthesis by organisms have not yet been conclusively elucidated. The most widely accepted mechanism for the extracellular biosynthesis of copper nanoparticles involves the extracellular nitrate reductase present in culture supernatants that helps transfer electrons to and hence reduce silver ions to metallic copper. ${ }^{7,8}$ However other active biomolecules, such as, proteins, polysaccharides, and DNA, have also been suggested to be responsible for the synthesis of metal NPs in microbial systems. ${ }^{9,10}$ Studies on action of copper nanoparticles have shown that positive charge on metal ion is critical for its antimicrobial activity as it allows electrostatic interaction between negatively charged bacterial cell membranes and positively charged nanoparticles. It has also been proposed that there can be release of copper ions by the nanoparticles, and these ions can interact with the thiol groups of many vital enzymes and inactivate them. Copper ions contact with bacterial cell which inhibit the different functions in the cell and damage the cells. ${ }^{11,12}$ Another reason is that the DNA has phosphorus and sulfur as main components; the nanoparticles react with soft bases and destroy the DNA and finally lead to cell death. The interaction of the silver nanoparticles with the sulfur and phosphorus of the DNA can lead to problems in the DNA replication of the bacteria and thus terminate the microbes. ${ }^{3,13}$

In present study, the copper nanoparticles were coated on the surface of water purification filter using a biological technique. The coating characteristics were investigated using X-ray diffraction (XRD), transmission electron microscopy (TEM), Uv-vis spectroscopy and atomic absorption spectroscopy (AAS). The main purpose is the product of copper nanoparticles by biological method under optimal conditions and then its coating on the filter. In addition, the antimicrobial activity of the prepared filter was studied by measuring the diameter of the inhibition zone by disc diffusion method.

\section{RESULTS AND DISCUSSION}

\section{Coating of nanoparticles on the filter}

XRD analysis was used to confirm the presence of copper nanoparticles on the coated filter surface. As shown in Fig. 1, the presence of crystalline copper in the coated filter was confirmed by the XRD analysis. The observation of absorption peaks at angles $43.39^{\circ}, 50.55^{\circ}$ and $74.08^{\circ}$ indicated the presence of copper nanoparticles on the filter surface. ${ }^{10,11}$ Thus, XRD confirmed the synthesized copper nanoparticles were crystalline copper and had a face-centered cubic structure.

A number of strong Bragg reflections can be seen which correspond to the (111), (200) and (220) reflections of face centered cubic symmetry (FCC) copper. The XRD shows that copper nanoparticles formed are crystalline. Three peaks at $2 \theta$ values of $43.39^{\circ}, 50.55^{\circ}$ and $74.08^{\circ}$ corresponding to (111), (200) and (220) planes of copper is observed and compared with the standard powder diffraction card of Joint Committee on Powder Diffraction Standards (JCPDS), copper file N0. 04-0836. The experimental diffraction angle

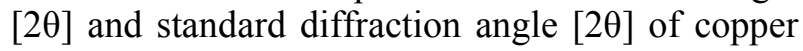
specimen in the Table 3 are in agreement.

\section{The effect of temperature on copper nanoparticles coating}

To evaluate the effect of temperature, three containers containing $100 \mathrm{~mL}$ of copper nitrate $(0.001 \mathrm{M})$ were maintained at three different temperatures of $5^{\circ} \mathrm{C}, 25^{\circ} \mathrm{C}, 50^{\circ} \mathrm{C}$. Transmission electron microscopy (TEM) was used to measure the size and shape of coated nanoparticles on the filter surface. Particle size is a fundamental property of nanoparticles because it influences the properties, such as, saturation solubility, dissolution speed, physical stability, and even biological performances ${ }^{14}$. The TEM analysis showed that the temperature was an effective factor on the size of coated copper nanoparticles, so that the size of the nanoparticles was about 35 $\mathrm{nm}, 55 \mathrm{~nm}$ and $95 \mathrm{~nm}$ at $5{ }^{\circ} \mathrm{C}, 25^{\circ} \mathrm{C}$ and $50{ }^{\circ} \mathrm{C}$, respectively. Therefore, temperature optimum for copper nanoparticles coating on filter was $5{ }^{\circ} \mathrm{C}$ due to the size of the copper nanoparticles is smaller and its distribution is narrower (Figs. 2 to 4 ). 


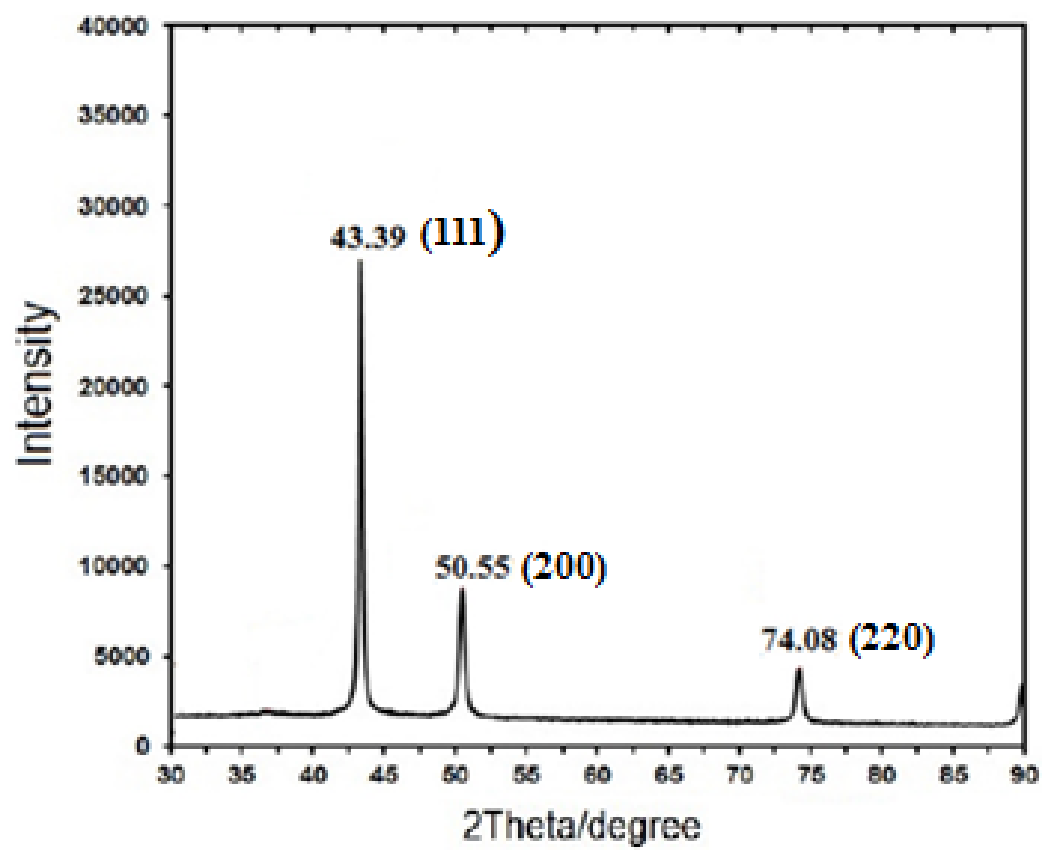

Fig. 1 - XRD patterns of the copper nanoparticles coated on cotton filter.

\section{Table 1}

Experimental and standard diffraction angles of copper specimen

\begin{tabular}{c|c}
\hline $\begin{array}{c}\text { Experimental diffraction angle } \\
{[\mathbf{2 \theta} \text { in degrees] }}\end{array}$ & $\begin{array}{c}\text { Standard diffraction angle } \\
\text { [20 in degrees] JCPDS Copper: 04-0836 }\end{array}$ \\
\hline $43.39^{\circ}$ & $43.297^{\circ}$ \\
$50.55^{\circ}$ & $50.433^{\circ}$ \\
$74.08^{\circ}$ & $74.130^{\circ}$ \\
\hline
\end{tabular}

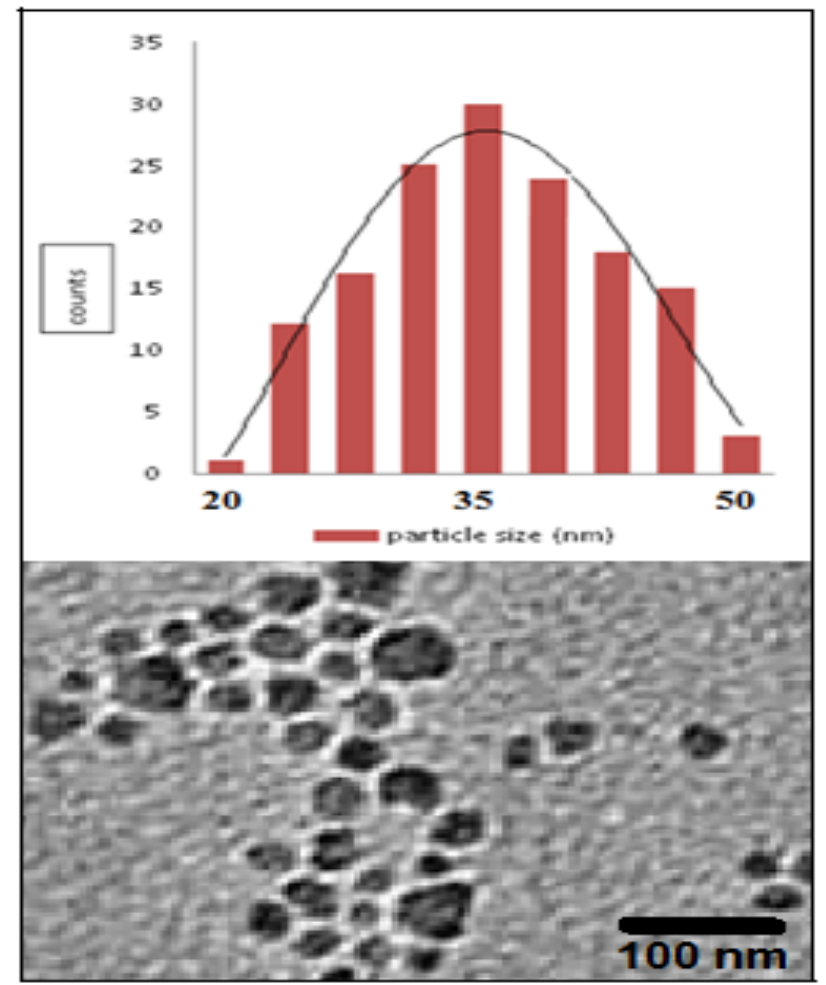

Fig. 2 - TEM image of the copper nanoparticles coated on cotton filter at $5^{\circ} \mathrm{C}$. 


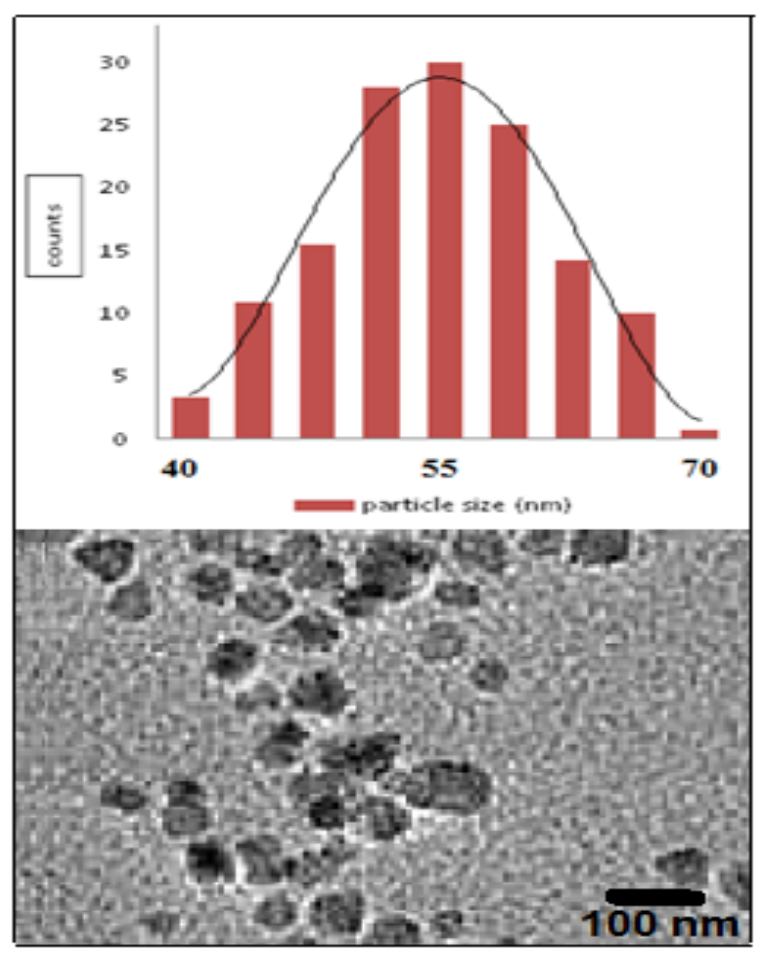

Fig. 3 - TEM image of the copper nanoparticles coated on cotton filter at $25^{\circ} \mathrm{C}$.

\section{The release amount of copper ion from coated filter}

To investigate the release of copper ion, the coated filter was placed in distilled water. Then, 10 $\mathrm{ml}$ of the water was tested after one day, one week and one month by atomic absorption spectroscopy (AAS) to measure copper ion concentration. The copper ions were released from the filter in distilled water, $0.02 \mathrm{ppm}$ after one day, $0.04 \mathrm{ppm}$ after one week, and $0.05 \mathrm{ppm}$ after a month. It seems coated filter is suitable for use in water treatment due to appropriate coating of copper nanoparticles on cotton filter.

\section{The study of antibacterial activity}

The antibacterial activities of coated filter against various pathogenic microorganisms, including Gram-negative and positive bacteria were investigated. Results are presented in Tables 2 and 3. The diameters of inhibition zones for Gram-negative bacteria (E. Coli) were $3.6 \mathrm{~mm}$ and for Gram-positive bacteria (S. aureus) was $4.2 \mathrm{~mm}$. Antibacterial activity of the coated filter against two bacteria E. coli and S. aureus was lesser than tetracycline antibiotic, while compared to the cephalexin antibiotic showed a higher

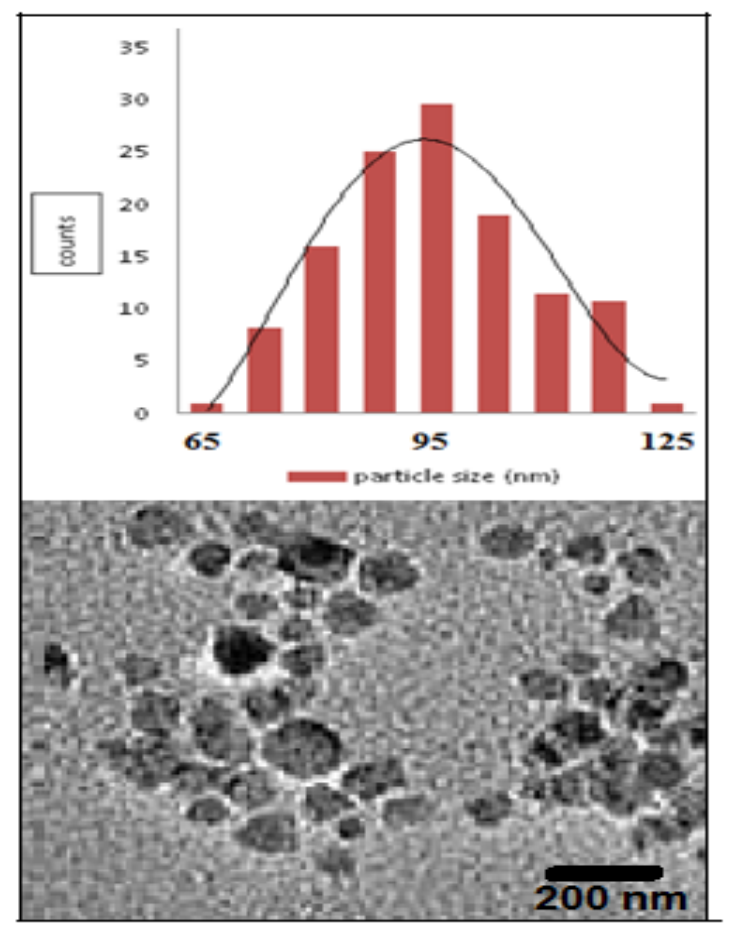

Fig. 4 - TEM image of the copper nanoparticles coated on cotton filter at $50^{\circ} \mathrm{C}$.

antimicrobial activity. As indicated in the tables, the antibacterial activity of the coated filter against $S$. aureus is greater than E. coli. In addition, it was determined that coated filter exhibited an appropriate antibacterial activity against these two bacteria. Previous results showed that $S$. aureus presented the highest sensitivity to copper nanoparticles, whereas E. coli was less sensitive., ${ }^{2,4}$ Hence our results were in agreement with these reports, and they demonstrated the bactericidal effect of the coted filter.

Table 2

Antibacterial activity of coated filter on $S$. aureus (mm)

\begin{tabular}{c|c}
\hline Samples & Zone of inhibition (mm) \\
\hline control (cotton filter) & 0 \\
copper nanoparticles coated & 4.2 \\
filter & \\
tetracycline & 8.5 \\
cefalexin & 0 \\
\hline
\end{tabular}

Table 3

Antibacterial activity of coated filter on E. Coli (mm)

\begin{tabular}{c|c}
\hline Samples & Zone of inhibition (mm) \\
\hline control (cotton filter) & 0 \\
copper nanoparticles coated & 3.6 \\
filter & 5.7 \\
tetracycline & 0 \\
cefalexin & \\
\hline
\end{tabular}




\section{EXPERIMENTAL}

The filter used in this research was a cotton filter. The filter was coated with copper nanoparticles using a biological method. E. Coli was inoculated into flasks containing sterile Tryptic Soy Broth (TSB) and the flasks were incubated at $37^{\circ} \mathrm{C}$ for a day. After the incubation period the culture was centrifuged at $4500 \mathrm{rpm}$ for $25 \mathrm{~min}$ and the supernatant was applied for the next steps. Using the results of the past studies, the copper nanoparticles were coated at a concentration of $0.001 \mathrm{M}$ of copper nitrate. The concentration of $0.001 \mathrm{M}$ was an optimum concentration to form copper nanoparticles ${ }^{14}$.

\section{Studying the coating}

\section{of copper nanoparticles on cotton filter}

A container containing $100 \mathrm{~mL}$ of copper nitrate $(0.001$ M) was prepared. In container bottom, the cotton filter was fixed. Then, $10 \mathrm{cc}$ supernatant was added to this container and the color change of copper nitrate solution was accomplished after about two hours. ${ }^{14}$ The synthesized copper nanoparticles deposited on the cotton filter. It was used from Padder padded press (Super Mini Pad Tabletop Paper Padding Press) to stabilize nanoparticles on the filter surface. Filter coated with copper nanoparticles was stabilized by pressing between two pads. XRD analysis was used to prove the nanoparticles on the filter. X-ray diffraction (XRD) analysis is an analytical technique designed to provide more in-depth information about crystalline compounds, including identification and quantification of crystalline phases. This is a useful tool when trying to positively identify a contaminant or corrosion product, and for identification of foreign phases for purity analyses of crystalline powders.

\section{The effect of temperature on the coating of copper nanoparticles on cotton filter}

To study the effect of temperature, three containers containing $100 \mathrm{~mL}$ of copper nitrate $(0.001 \mathrm{M})$ were maintained at three different temperatures of $5^{\circ} \mathrm{C}, 25^{\circ} \mathrm{C}, 50^{\circ} \mathrm{C}$. The cotton filter was fixed in container bottom. Then, $10 \mathrm{~mL}$ supernatant were added to this container and the color change of copper nitrate solution was accomplished after about two hours. It was used from Padder padded press (Super Mini Pad Tabletop Paper Padding Press) to stabilize nanoparticles on the filter surface. Filter coated with copper nanoparticles was stabilized by pressing between two pads. To observe the shape and size of copper nanoparticles on filter, transmission electron microscopy (TEM) was used. Transmission electron microscopy (TEM) is a microscopy technique in which a beam of electrons is transmitted through a specimen to form an image. The specimen is most often an ultrathin section less than $100 \mathrm{~nm}$ thick or a suspension on a grid. An image is formed from the interaction of the electrons with the sample as the beam is transmitted through the specimen.

\section{Studying the release of copper ion from coated filter}

In this step, coated filter was put into distilled water to specify the release amount of copper ion from filter. Then, 10 $\mathrm{mL}$ of the water was tested after one day, one week and one month by atomic absorption spectroscopy (AAS) to measure copper ion concentration. Atomic Absorption Spectroscopy (AAS) is a spectroscopic method for measuring metals concentration with a detection limit below $1 \mathrm{ppm}$. It acts on the basis of the absorbance spectrum of sample. Due to its high sensitivity, it can be used to measure toxic metals in drinking water.

\section{Studying the antibacterial activity of coated filter}

The antibacterial activity of coated filter was investigated against two bacteria of Escherichia coli and Staphylococcus aureus. It was used disc diffusion technique to determine the antibacterial activity of the coated filter. ${ }^{9,14}$ In present work, agar plates are inoculated with two bacteria of $E$. coli and $S$. aureus. Then, two antibiotics, a coated filter and a cotton filter (uncoated) as control sample were placed on the agar surface. The Petri dishes were incubated at $37^{\circ} \mathrm{C}$ for $24 \mathrm{hr}$. Finally, antibacterial agent diffused into the agar and inhibited growth of two bacteria and then the diameters of inhibition growth zones were measured.

\section{CONCLUSIONS}

The main purpose of this research was the product of antibacterial filters for water purification. In this work, the copper nanoparticles were synthesized by E. Coli and then coated on a filter. It was confirmed the existence of the copper nanoparticles on the coated filter by XRD. Also, it was determined a temperature of $5{ }^{\circ} \mathrm{C}$ as the optimum temperature of coating process using TEM. In addition, the antibacterial activity of the coated filter was investigated by disc diffusion method against two bacteria E. Coli and S. Aureus. It seems the biological methods are suitable for nanoparticles coating on filters or other materials such as membrane and paper according to the obtained results of this study. This copper nanoparticles coated filter has the potential to become an extremely low-cost way to purify water. This is especially suitable for resource limited countries.

\section{REFERENCES}

1. L. Wei, J. Lu, H. Xu, A. Patel, Z. S. Chen and G. Chen, Drug Discov. Today, 2015, 20, 595-601.

2. J. P. Ruparelia, A. K. Chatterjee, S. P. Duttagupta and S. Mukherji, Acta Biomater. 2008, 4, 707-716.

3. H. R. Ghorbani, Chem. Eng. Commun., 2015, 202, $1463-$ 1467

4. M. Raffi, S. Mehrwan, T. M. Bhatti, J. I. Akhter, A. H. Ameed, W. Yawar and M. M. ul Hasan, Ann. Microbiol., 2010, 60, 75-80.

5. A. K. Chatterjee, R. K. Sarkar, A. P. Chattopadhyay, P. Aich, R. Chakraborty and T. Basu, Nanotechnol., 2012, 23, 1-11.

6. Y. Wei, S. Chen, B. Kowalczyk, S. Huda, T. P. Gray and B. A. Grzybowski, J. Phys. Chem C., 2010, 114, 15612 15616.

7. W. Shao, S. Wang, J. Wu, M. Huang, H. Liu and H. Min, RSC Adv., 2016, 6, 65879-65884.

8. J. Ramyadevi, K. Jeyasubramanian, A. Marikani, G. Rajakumar and A. A. Rahuman, Mater. Lett., 2012, $71,114-116$. 
9. H. R. Ghorbani, Minerva Biotechnol., 2016, 28, 86-88.

10. S. Chandra, A. Kumar and P. K. Tomar, J. Saudi Chem. Soc., 2014, 18, 149-153.

11. D. G. Deryabin, E. S. Aleshina, A. S. Vasilchenko, T. D. Deryabina, L. V. Efremova, I. F. Karimov and L. B. Korolevskaya, Nanotechnol. Russia, 2013, 8, 402-408.
12. R. Bürgers, A. Eidt and R. Frankenberger, Arch. Oral Biol., 2009, 54, 595-601.

13. T. Y. Klein, J. Wehling, and L. Treccani, Environ. Sci. Technol., 2013, 47, 1065-1072.

14. M. Haghighi and H. R. Ghorbani, Nanomed. J., 2018, 5, 90-95. 